\title{
Reducing the Field of View in Correlating Wavefront Sensors for Solar Adaptive Optics
}

\author{
Matthew J. Townson ${ }^{\mathrm{a}}$ and Christopher D. Saunter ${ }^{\mathrm{a}}$ \\ ${ }^{a}$ Dept. Physics, Durham University, South Road, Durham, DH1 3LE, UK
}

\begin{abstract}
Wavefront sensing for solar adaptive optics currently requires the use of extended-field Shack-Hartmann wavefront sensors. Such wavefront sensors suffer from a reduced sensitivity to high altitude turbulence due effects of measuring wavefront gradients using the relative motion within the extended-field. We discuss the performance of centroiding techniques in a correlation Shack-Hartmann wavefront sensor and the insensitivity to high altitude turbulence and propose a method for reducing the field of view required to effectively measure the wavefront gradient in order to increase the sensors sensitivity to high altitude turbulence.
\end{abstract}

Keywords: Solar Adaptive Optics, Wavefront Sensing, Shack-Hartmann

\section{INTRODUCTION}

In Solar Adaptive Optics (AO) the Wavefront Sensor (WFS) typically employed are Shack-Hartmann Wavefront Sensors (SH-WFSs). ${ }^{1-3}$ This choice is driven by the ability of the SH-WFS to measure wavefront gradients while observing extended sources. When observing an extended Field of View (FOV) a SH-WFS forms a series of images of the extended object in the focal plane of the WFS. These images are displaced relative to each-other in a similar way to the displacement of the spots formed by a SH-WFS observing a point source.

In order to measure the local wavefront gradient across a sub-aperture in the WFS the local displacement of the image formed needs to be measured. A cross-correlation can be taken between the image formed in a subaperture and a reference image, then the resulting correlation image can be centroided to measure the location of the correlation peak. The displacement of the peak of the correlation image corresponds to the displacement between the reference image and the sub-aperture image and hence the local wavefront gradient.

Observing an extended-field in a SH-WFS introduces an effect which reduces the WFSs sensitivity to high altitude turbulence. ${ }^{4,5}$ This is due to the increasing size of the sub-aperture meta-pupil as a function of altitude. At higher altitudes the effective sub-aperture pupil size is larger, so perturbations of the wavefront at altitude are sampled at a coarser scale than at the ground. ${ }^{2,6}$ Here we describe a potential method for reducing the size of the FOV required in an extended-field SH-WFS, which will reduce this effect. We suggest that reducing the FOV of the sub-apertures in the WFS, whilst retaining a larger FOV in the reference image allows for accurate centroids to be obtained for a sub-aperture for smaller FOVs than if the sub-aperture and reference image had a similar FOV.

The method was developed, and the results shown here derived from end-to-end simulations of solar SH-WFSs through a simplified turbulent profile consisting of a single high altitude layer $(10 \mathrm{~km})$ and a single layer located at the ground. Though un-realistic this profile includes both low and high layer turbulence to demonstrate the methods performance and ability to sense high layer turbulence. The simulations were made using Durham Adaptive Optics Simulation Platform (DASP) ${ }^{7}$ and subsequent data reduced using iPython ${ }^{8}$ and the Python programming language, using numpy ${ }^{9}$ and astropy ${ }^{10}$ libraries. Plots were created using the matplotlib ${ }^{11}$ plotting library.

M.J.T.: E-mail: matthew.townson@durham.ac.uk 


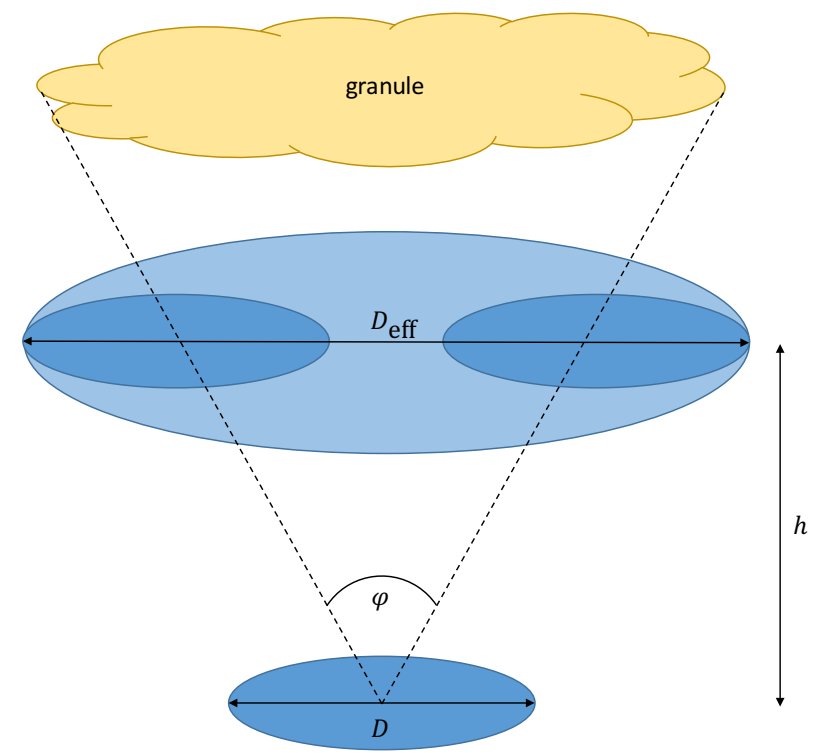

Figure 1. Due to the extended FOV observed, $\varphi$, the projected size of a pupil with diameter $D$ increases with increasing altitude. This creates a meta-pupil which is a function of altitude, changing the response of the sub-aperture to also become a function of altitude

\section{EXPANDING META-PUPIL FROM EXTENDED SOURCES}

Measuring the local wavefront gradient observing an extended FOV in a SH-WFS changes the geometry of the pupil to become a function of altitude ${ }^{2,6}$ as described in equation 1 ;

$$
D_{\text {eff }}=D+\varphi \times h,
$$

where $D$ is the diameter of the pupil, $\varphi$ is the angular size of the extended field in radians, and $h$ is the altitude of the plane where $D_{\text {eff }}$ is being calculated for in meters. The image formed by a single sub-aperture is affected by turbulence at all altitudes, causing global motion of the image as well as higher order aberrations within the sub-aperture image. When performing the cross-correlation of the sub-aperture image with a reference image and subsequently centroiding the correlation image the only the global motion of the sub-aperture image is measured. Using this to estimate the local wavefront gradient assumes that the entire FOV within the sub-aperture was affected by the same turbulence. However, this is not necessarily the case, as illustrated in Fig. 1, where different regions of the FOV of the sub-aperture trace different paths through the atmosphere.

The physical separation of the different field directions is a function of altitude, which changes the response of the WFS to become altitude dependant. The separation of the edges of the FOV increases with increasing altitude, creating a larger meta-pupil at larger altitudes. The local gradient measured within a sub-aperture from high altitude turbulence will be measured over this larger meta-pupil, so will be reduced in strength.

\section{EFFECT OF THE SIZE OF FIELD OF VIEW}

From Eqn. 1 the size of the FOV used in the cross-correlation for the extended-field SH-WFS affects the size of $D_{\text {eff. A larger FOV increases }} D_{\text {eff }}$ at a given altitude, whereas a smaller FOV decreases $D_{\text {eff }}$ for a given altitude. So, for a narrow FOV, the expansion of $D_{\text {eff }}$ with altitude can be minimised. This decrease in the meta-pupil diameter will increase the sensitivity of the WFS to high altitude turbulence. However, reducing the FOV in the sub-aperture images affects the accuracy of centroid measurements.

For a cross-correlation to create a peak with a high Signal to Noise Ratio (SNR) which can be accurately centroided there needs to be discernible features in the images being cross-correlated. For SH-WFS observing 


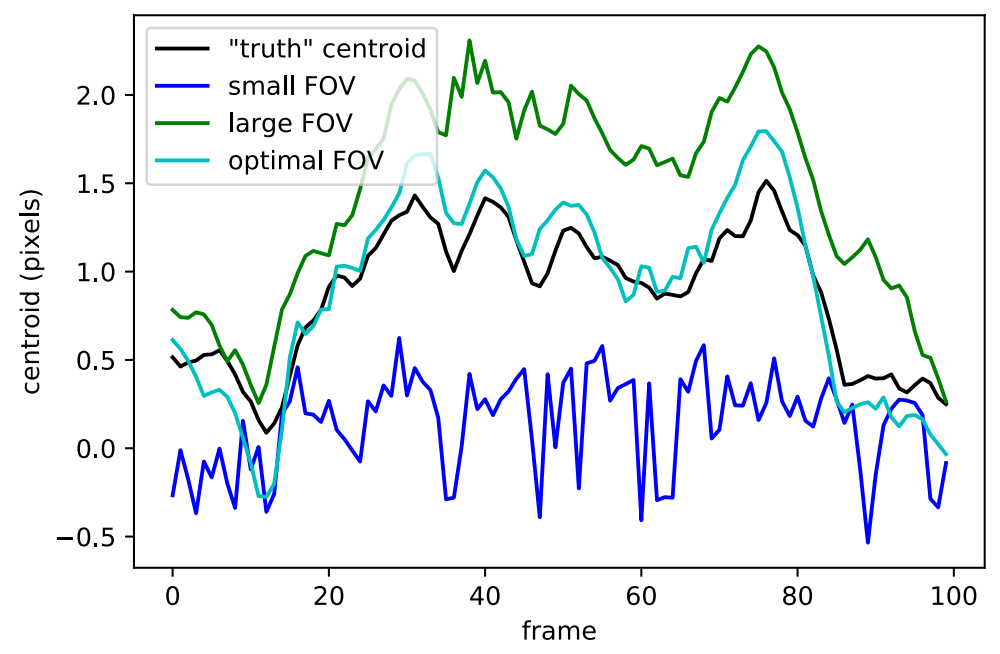

Figure 2. Example simulated SH-WFS with the known "truth" centroids corresponding to the on-axis centroids and correlation centroids using different FOVs. The black line shows the "truth" centroids, as would be measured from an on-axis point source, the blue shows a small FOV used in the cross-correlation centroid measurements (11 pixels) and the green shows a large FOV used in the cross-correlation centroid measurement (29 pixels). The cyan line shows the centroid estimates when using the "optimal" FOV in the cross-correlation (17 pixels).

the sun the most typical feature which is observed is solar granulation. ${ }^{1,12}$ Solar granulation has a typical size of $2-3^{\prime \prime}$. A FOV is required which incorporates multiple granules in order to most accurately measure the displacement between images using the cross-correlation technique. This results in a typical FOV for Solar SH-WFS of $\sim 10^{\prime \prime} .{ }^{13}$ Such a FOV has the effect of increasing a $1 \mathrm{~m}$ aperture at the ground to $1.5 \mathrm{~m}$ at $10 \mathrm{~km}$, an increase of diameter of $50 \%$.

An example set of image shifts, along with centroids from different FOV cross-correlations are shown in Fig. 2. This shows the effect of a small FOV used in the cross-correlation shown in blue, a large FOV shown in green and the "optimal" FOV shown in cyan alongside the shifts applied to the images in black. When the FOV is small there is not enough structure in the individual images for the cross-correlation to create a peak with enough SNR to allow for an accurate centroid to be measured. When the FOV is large the estimates follow the correct trend, however, the centroid estimates don't include the contribution from high altitude turbulence, giving an error. The optimal FOV is a compromise between the centroiding noise from a small FOV and reducing the sensitivity to high altitude turbulence by using a large FOV.

The errors from using different sized FOV in the cross-correlation correlation is shown in Fig. 3. Here we see a similar result to Fig. 2, for very small FOVs the error on the centroids is large. This then drops when there is enough structure in the individual sub-aperture images to generate cross-correlation images with a high SNR. However, as the FOV increases further the high altitude turbulence is increasingly attenuated, resulting in an increase in centroiding error for increasing FOV.

The optimal FOV can be estimated by using the technique described in Ref. 14 to estimate the error for different sized FOVs. This allows for an AO system which uses correlation SH-WFS observing extended objects to use the optimal FOV and calculate the most accurate centroids from sub-aperture and reference images with the same size FOV.

\section{REDUCING THE FIELD OF VIEW}

To reduce the impact of the expanding meta-pupil effect we propose to use a FOV in the sub-aperture images which is smaller than the FOV of the reference image. This allows for a reduction in the FOV in the subapertures, reducing the rate of the meta-pupil expansion as a function of altitude, but keeping the full structure 


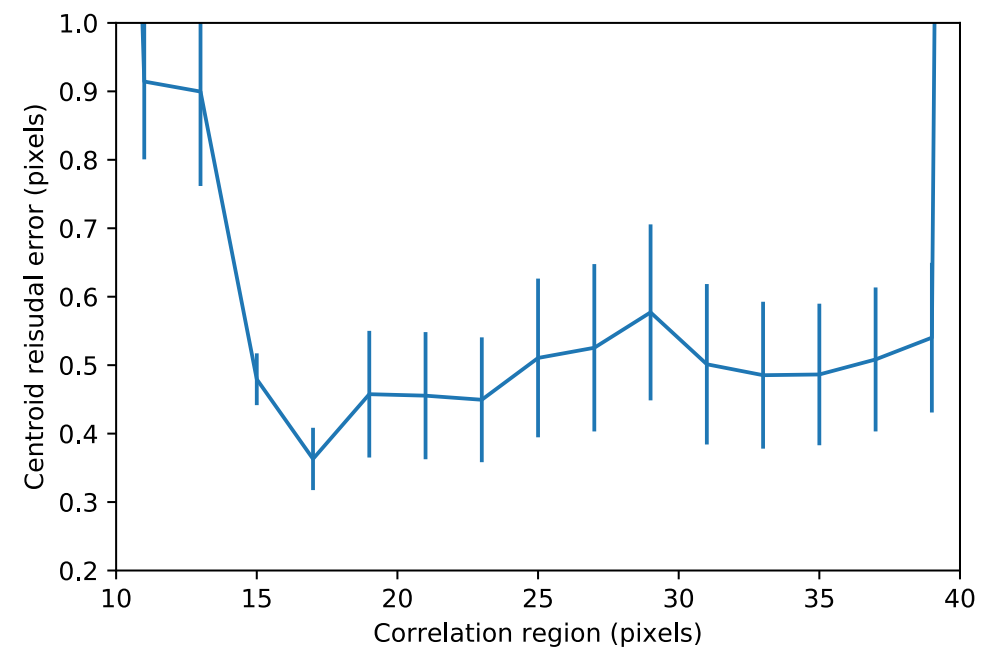

Figure 3. Centroiding error for different sized cross-correlation regions. For both large and small FOVs the residual centroiding error is large. However, there is a well in the middle region of FOVs where the error error is slowly increasing. This slow increase in centroiding error is from the attenuation of the high altitude turbulence with the increasing FOV in the cross-correlation.

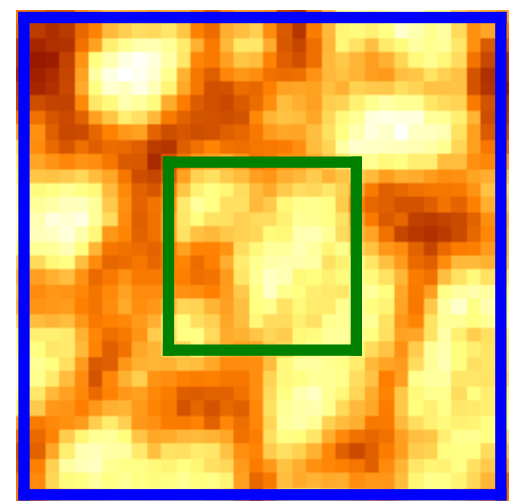

Figure 4. Example differential FOVs for sub-aperture and reference images in a correlation SH-WFS. The reference image FOV is depicted as the area inside the blue box and the sub-aperture FOV is depicted as the area inside the green box. By using two different size FOVs the full structure in the image is included in the reference image for the cross-correlation and the effect of the expanding meta-pupil minimised as the FOV of the sub-aperture is reduced.

in the reference image, allowing for accurate centroids to still be measured. A simple example of such a WFSing system is shown in Fig. 4.

Cross-correlating the larger reference image with smaller sub-aperture images can be implemented by padding the sub-aperture image to the size of the reference image, then generating the cross-correlation images using the traditional Fast Fourier Transform (FFT) or the square difference function, as suggested by Ref. 15. When padding the sub-aperture image to the size of the reference image it is important to minimise the introduction of any hard edges to the image structure which could introduce artefacts into the cross-correlation image and affect the centroid measurements. Images of solar granulation have a low contrast, ${ }^{16}$ typically of $10 \%$, meaning padding the sub-aperture images with zeros introduces structure with greater strength than the structure contained in the image of around $\sim 90 \%$. This effect can be minimised by using the median of the image as the intensity value for the padded elements, this reduces the difference in intensity from $\sim 90 \%$ to $\sim 5 \%$, leaving the structure contained in the sub-aperture images as the strongest component.

For a single SH-WFS which implements a smaller FOV in sub-aperture images an image with a larger FOV is 
not necessarily available to be used as a reference image in the cross-correlation. An artificial reference image can be generated from the SH-WFS images as the relative image shifts induced by the atmospheric turbulence cause different sub-apertures to sample different regions of the larger solar structure. These relatively shifted images can be combined using a shift-and-add technique if the relative shifts are known. In a working AO loop the relative shifts are measured by the WFS, allowing for the construction of the artificial reference image. However, this is not the case for generating an initial reference image to be used before the AO loop is closed. In this regime a method is required to measure the centroids when the sub-aperture images have large relative shifts which may not be accurately centroided by simply using a single sub-aperture image as the reference image.

\section{MEASURING CENTROIDS WITH LARGE RELATIVE SHIFTS}

In order to generate an artificial reference image centroids are required so the shift-and-add technique can be used to combine the individual sub-aperture images. Generating an initial set of centroid estimates to create an artificial reference image require the ability to centroid large relative shifts between sub-aperture images. In order to mitigate this problem we can consider the entirety of a SH-WFS frame together. The region of turbulence that the WFS samples is continuous, as is the gradient of the turbulence. ${ }^{17}$ Atmospheric turbulence structure also follows a power law which places most of the power of the turbulence at low orders. ${ }^{17,18}$ As SH-WFSs directly measure the gradient of the phase, adjacent sub-apertures should be subjected to a similar wavefront gradient. This will result in adjacent sub-apertures typically being shifted by similar amounts. So, in a single WFS frame there will be typically at least one sub-aperture with a similar absolute shift to any given sub-aperture, allowing for their relative displacement to be measured.

Using similar pairs of sub-apertures to estimate relative shifts can be used to measure centroids for all subapertures across the WFS. This can be posed as a least squares minimisation on the centroids where the centroid between all possible pairs of sub-apertures, $i$ and $j$ are measured. This least squares problem can be weighted by the inverse of the magnitude of the centroid which is measured, weighting towards pairs of sub-apertures with small relative shifts. This weight factor can be expressed as;

$$
w^{i, j}=\frac{1}{\mathbf{R}^{i, j}},
$$

where $w^{i, j}$ is the weight applied to the centroid measurement $\mathbf{R}^{i, j}$, which is the relative shift between subapertures $i$ and $j$. The inclusion of a weight in the measured centroids creates a least squares problem of the form;

$$
\left(\begin{array}{ccc}
w^{0,1} & -w^{0,1} & \\
w^{0,2} & & -w^{0,2} \\
& w^{1,2} & -w^{1,2}
\end{array}\right)\left(\begin{array}{l}
\mathbf{R}^{0} \\
\mathbf{R}^{1} \\
\mathbf{R}^{2}
\end{array}\right)=\left(\begin{array}{c}
w^{0,1} \\
w^{0,2} \\
w^{1,2}
\end{array}\right)\left(\begin{array}{l}
\mathbf{R}^{0,1} \\
\mathbf{R}^{0,2} \\
\mathbf{R}^{1,2}
\end{array}\right)^{T},
$$

for an example WFS with only three sub-apertures. This can then be solved for the individual sub-aperture shifts $\mathbf{R}^{i}$. Once a set of initial centroids have been obtained an initial reference image can be generated using a shift-and-add technique with the individual sub-aperture images and their shifts. However, the resulting generated reference image is likely to have a low SNR. Subsequent artificial reference images can be generated using a bootstrap method to update the generated reference image by combining the SH-WFS images each iteration using the new centroid measurements. Once a final reference image is generated they can be updated at intervals by using the centroid measurements from the AO system and the sub-aperture images which the centroids correspond to.

\section{CONCLUSIONS}

Using an extended FOV in a correlation SH-WFS leads to attenuation of high altitude turbulence. We proposed a method for reducing this effect by using a larger FOV in the reference image than in the sub-aperture images. This allows for the FOV in the sub-aperture images to be reduced. The reduction in the FOV of the sub-aperture images should decrease the attenuation of the high altitude turbulence, as the meta-pupil expansion is reduced, allowing both accurate centroid measurements and also increasing the sensitivity of tomographic AO systems 
which use this method. We also described a method for measuring centroids where the images have large relative shifts and generating artificial reference images from the images and centroids.

The effect of this centroiding technique on AO systems is currently being investigated. Further future work for increasing the sensitivity of correlation SH-WFS to high altitude turbulence, include exploring further the effects of padding individual sub-aperture images in the cross-correlation process.

\section{ACKNOWLEDGMENTS}

MJT acknowledges support for part of this work from the Science and Technology Facilities Council (STFC) in the form of a PhD studentship (ST/K501979/1). The authors would also like to thank Alastair Basden for his advice and help with using the DASP simulation package.

\section{REFERENCES}

[1] Scharmer, G. B., Dettori, P., Löfdahl, M. G., and Shand, M., "Adaptive optics system for the new Swedish solar telescope," Proc. SPIE 4853, 370-380 (2003).

[2] Berkefeld, T., Soltau, D., del Moro, D., and Löfdahl, M., "Wavefront Sensing and Wavefront Reconstruction for the 4m European Solar Telescope EST," Proc. SPIE 7736, 77362J-77362J-9 (7 2010).

[3] Richards, K., Rimmele, T., Hegwer, S. L., Upton, R. S., Woeger, F., Marino, J., Gregory, S., and Goodrich, B., "The Adaptive Optics and Wavefront Correction Systems for the Advanced Technology Solar Telescope," Proc. SPIE 7736, 773608-773608 (7 2010).

[4] Marino, J., "Expected performance of solar adaptive optics in large-aperture solar telescopes," Optical Engineering 51(10), 101709 (2012).

[5] Tallon, M., Montilla, I., Langlois, M., Lyon, O. D., Laval, S.-g., and Lyon, R. A. D., "Wide-field wavefront sensing in solar adaptive optics: modeling and effects on reconstruction," Third AO4ELT Conference (May), 1-8 (2013).

[6] Montilla, I., Tallon, M., Langlois, M., Béchet, C., and Collados Vera, M., "Modeling the effect of high altitude turbulence in wide-field correlating wavefront sensing and its impact on the performance of solar AO systems," Ao4Elt4 9148, 91486D (8 2014).

[7] Basden, A., Butterley, T., Myers, R., and Wilson, R., "Durham extremely large telescope adaptive optics simulation platform.," Applied Optics 46(7), 1089-1098 (2007).

[8] Pérez, F. and Granger, B. E., "IPython: A System for Interactive Scientific Computing," Computing in Science \& Engineering 9, 21-29 (2007).

[9] Van der walt, S., Colbert, S. C., and Gaël, V., "The NumPy array: a structure for efficient numerical computation," Computing in Science \& Engineering 13, 22-30 (2011).

[10] Robitaille, T. P., Tollerud, E. J., Greenfield, P., Droettboom, M., Bray, E., Aldcroft, T., Davis, M., Ginsburg, A., Price-Whelan, A. M., Kerzendorf, W. E., Conley, A., Crighton, N., Barbary, K., Muna, D., Ferguson, H., Grollier, F., Parikh, M. M., Nair, P. H., Günther, H. M., Deil, C., Woillez, J., Conseil, S., Kramer, R., Turner, J. E. H., Singer, L., Fox, R., Weaver, B. A., Zabalza, V., Edwards, Z. I., Azalee Bostroem, K., Burke, D. J., Casey, A. R., Crawford, S. M., Dencheva, N., Ely, J., Jenness, T., Labrie, K., Lim, P. L., Pierfederici, F., Pontzen, A., Ptak, A., Refsdal, B., Servillat, M., and Streicher, O., "Astropy: A community Python package for astronomy," A \& $A$ 558, A33 (2013).

[11] Hunter, J. D., "Matplotlib: A 2D graphics environment," Computing in Science and Engineering 9(3), 99-104 (2007).

[12] Marino, J., "Expected performance of solar adaptive optics in large-aperture solar telescopes," Optical Engineering 51(10), 101709 (2012).

[13] Montilla, I., Marino, J., Asensio Ramos, A., Collados, M., Montoya, L., and Tallon, M., "Solar adaptive optics: specificities, lessons learned, and open alternatives," Proc. SPIE 9909, 99091H (2016).

[14] Townson, M. J., Saunter, C. D., and Kellerer, A., "Improved shift estimates on extended Shack-Hartmann wave-front sensor images," MNRAS 7(April), 1-7 (2015).

[15] Löfdahl, M. G., "Evaluation of image-shift measurement algorithms for solar Shack-Hartmann wavefront sensors," A \& A 524, A90 (11 2010). 
[16] Rimmele, T. R. and Radick, R. R., "Solar Adaptive Optics at the National Solar Observatory," Proc. SPIE 3353(March), 72-81 (1998).

[17] Hardy, J. W., [Adaptive Optics for Astronomical Telescopes], Oxford University Press (1998).

[18] Klyatskin, V. and Tatarskii, V. I., "Statistical Therory of light propagation in a turbulent medium," Izvestiya Vysshikh Uchebnykh Zavedenii, Radiofizika 15(10), 1433-1455 (1972). 